\title{
On Use of Multi-Chambered Fission Detectors for In-Core, Neutron Spectroscopy
}

\author{
Jeremy A. Roberts
}

\begin{abstract}
Presented is a short, computational study on the potential use of multichambered fission detectors for in-core, neutron spectroscopy. Motivated by the development of very small fission chambers at CEA in France and at Kansas State University in the U.S., it was assumed in this preliminary analysis that devices can be made small enough to avoid flux perturbations and that uncertainties related to measurements can be ignored. It was hypothesized that a sufficient number of chambers with unique reactants can act as a real-time, foilactivation experiment. An unfolding scheme based on maximizing (Shannon) entropy was used to produce a flux spectrum from detector signals that requires no prior information. To test the method, integral, detector responses were generated for singleisotope detectors of various $\mathrm{Th}, \mathrm{U}, \mathrm{Np}, \mathrm{Pu}, \mathrm{Am}$, and $\mathrm{Cs}$ isotopes using a simplified, pressurized-water reactor spectrum and fluxweighted, microscopic, fission cross sections, in the WIMS-69 multigroup format. An unfolded spectrum was found from subsets of these responses that had a maximum entropy while reproducing the responses considered and summing to one (that is, they were normalized). Several nuclide subsets were studied, and, as expected, the results indicate inclusion of more nuclides leads to better spectra but with diminishing improvements, with the best-case spectrum having an average, relative, group-wise error of approximately $51 \%$. Furthermore, spectra found from minimum-norm and Tihkonov-regularization inversion were of lower quality than the maximum entropy solutions. Finally, the addition of thermal-neutron filters (here, $\mathrm{Cd}$ and Gd) provided substantial improvement over unshielded responses alone. The results, as a whole, suggest that in-core, neutron spectroscopy is at least marginally feasible.
\end{abstract}

Index Terms-neutron spectroscopy, fission chambers, maximum entropy

\section{INTRODUCTION}

$\mathbf{I}$ $\mathrm{N}$ recent years, substantial interest has grown in the development of in-core instrumentation that can provide data for validation of advanced computational models for reactor analysis. Work at Commissariat à l'énergie atomique et aux énergies alternatives (CEA) [1] to produce "sub-miniature" fission chambers and at Kansas State University (KSU) [2], [3] to produce "micro-pocket" fission chambers has led to extremely small devices that provide opportunities for multiple, in-core flux monitors that can provide a detailed map of the core power over varying operational conditions. Some work has been done to determine continuous flux shapes from the point-wise measurements [4], and ongoing research aims to provide devices capable of exhibiting linear response across

This work was presented at the International Conference on Advancements in Nuclear Instrumentation Measurement Methods and their Applications, June 19-23, 2017.

J. Roberts is with the Department of Mechanical and Nuclear Engineering, Kansas State University, Manhattan, KS, 66502 (email: jaroberts@ksu.edu). many decades of power levels, e.g., as observed in pulsed reactors like the Transient Reactor Test Facility (TREAT) at Idaho National Laboratory and various university reactors, including the Training, Research, Isotopes, General Atomic (TRIGA) Mark II research reactor at KSU.

With their small size, it may be possible to use several, co-located sub-miniature or micro-pocket fission detectors to measure a neutron spectrum at a point. Even in the earliest developments at KSU, it was proposed to deploy two-chamber devices loaded with uranium and thorium, respectively, in order to distinguish between thermal and fast fluxes [2]. However, the threshold energy for thorium fission is approximately 1 $\mathrm{MeV}$, too large to provide sufficient detail in the epithermal region. Here, alternative reactants, in greater number, were explored to understand how the resulting signals from multiple chambers could be used to resolve the neutron spectrum. In particular, it was hypothesized that a sufficient number of chambers with unique reactants can act as a real-time, foilactivation experiment and that, with appropriate prior modeling and uncertainties, a Bayesian framework can be used to provide improved estimates for the energy spectrum. In this preliminary effort, no such prior information was assumed, leaving the full Bayesian framework for future work. Rather, the focus was placed squarely on understanding how much information can be extracted from an unknown spectrum via a set of integral responses. The key throughout was the presumed availability of devices small enough that several can be placed in a small region and that any perturbations to the local flux (magnitude or spectrum) are negligible.

\section{METHODS}

Shown in Fig. 1 are the microscopic fission cross sections for several nuclides of $\mathrm{Th}, \mathrm{U}, \mathrm{Np}, \mathrm{Pu}, \mathrm{Am}$, and $\mathrm{Cm}$. The values shown are evaluated at $300 \mathrm{~K}$. The same cross sections are shown in the 69-group WIMS [6] format condensed using the simplified, pressurized-water reactor (PWR) spectrum shown in Fig. 3. Observe that the spectrum used has no fine structure and, hence, represents a very simple test case for unfolding. On a related note, the geometry of any detector of interest will be sufficiently small (by assumption) that the infinite-dilution approximation is valid. In other words, self-shielding effects due to the detectors themselves are negligible and need not be accounted for in determination of the multigroup constants and, hence, the detector response functions.

Suppose an $N$-chamber device were produced having a sufficient mass of each reactant shown in Fig. 2 to produce a measurable, negligible-uncertainty signal in some application 


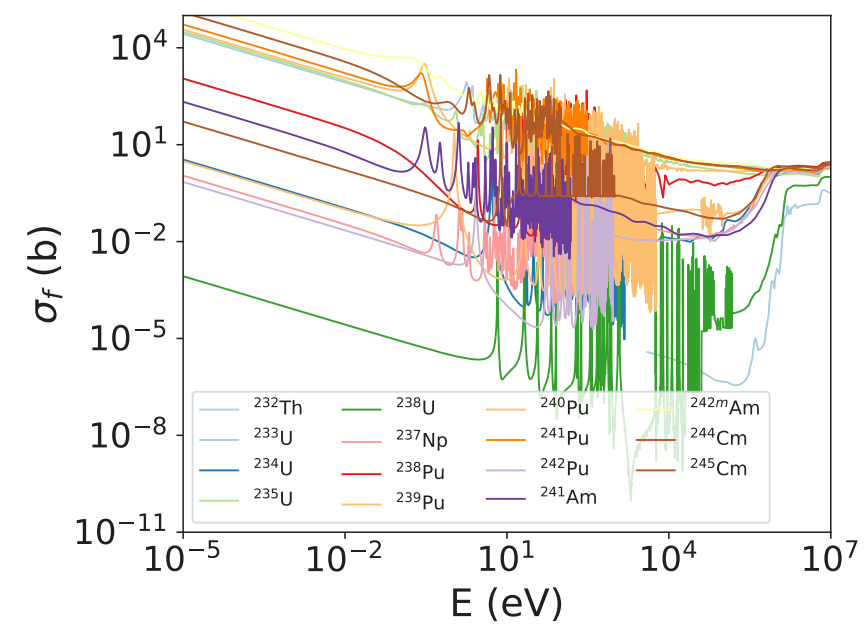

Fig. 1. Microscopic fission cross sections for several nuclides. All data was pulled directly from NNDC [5].

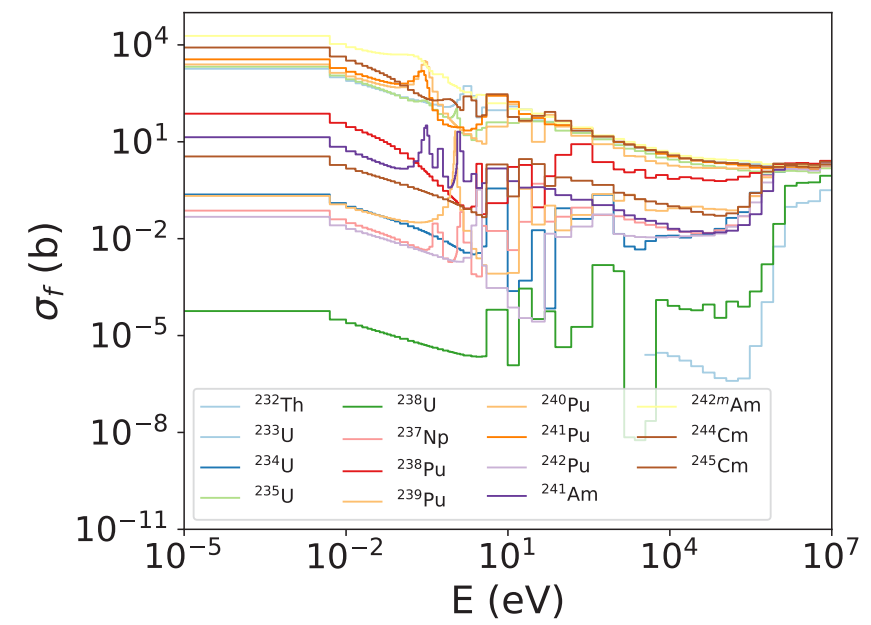

Fig. 2. Condensed microscropic, fission cross sections in the WIMS 69-group format.

reactor. Suppose further that the goal is to determine the flux spectrum of the application reactor using the 69-group WIMS energy structure. The resulting system of equations is

$$
r_{i}=\sum_{g=1}^{69} \Sigma_{f g, i} \phi_{g}, \quad i=1 \ldots N,
$$

or, in matrix form,

$$
\mathbf{r}=\boldsymbol{\Sigma}_{f} \phi
$$

where $\phi_{g}$ is the group-integrated flux, and it has been assumed that there are no losses due to dead time or discriminators. This is a major assumption with respect to the technology, but if the response of each device is linear, and if any losses are proportional to the signal similarly for all reactants, then the equations need only be modified by a single, multiplicative constant on the right. For real-world cases, further work must be done to include such features of the system explicitly.

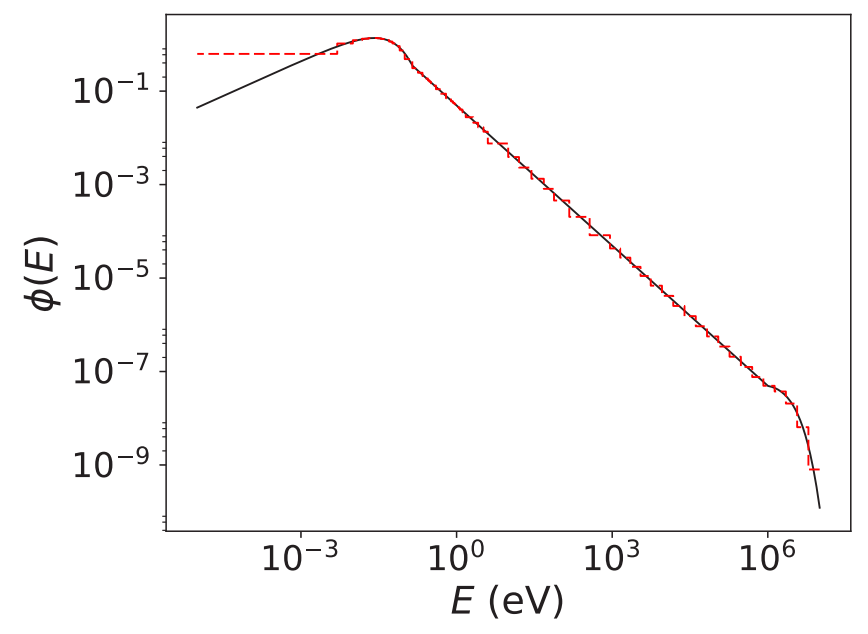

Fig. 3. Simplified, PWR-like spectrum used to weight cross sections as response functions; the multigroup equivalent is also shown.

Equation (1) is an underdetermined system, meaning that specialized techniques or additional constraints are needed. Such a problem belongs to the class of spectrum unfolding problems, itself a class of inverse problems. Many methods have been proposed to solve such problems, whether arising from Bonner-sphere spectroscopy or other applications. One simple inversion technique is to compute the minimum-norm solution, i.e.,

$$
\phi=\boldsymbol{\Sigma}\left[\boldsymbol{\Sigma} \boldsymbol{\Sigma}^{T}\right]^{-1} \mathbf{r},
$$

which yields a spectrum $\phi$ having the smallest Euclidean norm $|\phi|_{2}=\sqrt{\phi^{T} \phi}$. Although unique, the minimum-norm solution is not guaranteed to be strictly positive.

A second, straightforward inversion technique is Tihkonov regularization [7], i.e.,

$$
\phi=\left[\boldsymbol{\Sigma}^{T} \boldsymbol{\Sigma}+\mathbf{T}^{T} \mathbf{T}\right]^{-1} \boldsymbol{\Sigma} \mathbf{r},
$$

where a common regularization matrix is $\mathbf{T}=a \mathbf{I}$ for a scalar $a$ and identity matrix $\mathbf{I}$. Like the minimum-norm solution, the Tikhonov-regularization solution may also contain negative values.

For the work presented here, the primary method used is based on entropy maximization [8], in which the (Shannon) entropy of the spectrum is defined as

$$
S=-\sum_{g=1}^{69} \phi_{g} \ln \phi_{g} .
$$

The flux spectrum with the greatest entropy that satisfies both Eq. (1) and

$$
\sum_{g=1}^{69} \phi_{g}=1
$$

is found by solving the nonlinear, constrained optimization problem resulting from (1)-(6). Equation 6 implies that the integrated flux is known, which may not be the case in practice. However, the results below indicate that the unfolding process is not severely sensitive to the assumed, integral flux, meaning 
that even a crude estimate can be used. If the constraint is not employed, the results are much less favorable.

\section{RESULtS}

To demonstrate the use of entropy maximization for extracting spectral information from a set of unique, fissionchamber responses, a simple test problem was studied. In particular, the PWR spectrum shown in Fig. 3 was taken to be the application spectrum, with which reference response functions (here, just the microscopic, multigroup, fission cross sections) were defined. Then, spectra were unfolded for four base cases: (1) using only ${ }^{235} \mathrm{U},{ }^{238} \mathrm{U}$, and ${ }^{232} \mathrm{Th}$, (2) using the case- 1 nuclides in addition to ${ }^{237} \mathrm{~Np}$ and ${ }^{238} \mathrm{Pu}$, (3) using the case- 2 nuclides in addition to ${ }^{239} \mathrm{Pu},{ }^{240} \mathrm{Pu},{ }^{241} \mathrm{Pu}$, and ${ }^{242} \mathrm{Pu}$, and (4) using all nuclides shown in Fig. 2. Furthermore, case 3 was explored with and without the use of thermalneutron filters $\left({ }^{113} \mathrm{Cd}\right.$ and ${ }^{155} \mathrm{Gd}$ ), while case 4 was analyzed using minimum-norm and Thikonov-regularization inversion in addition to maximum-entropy inversion. ${ }^{1}$

The spectra resulting from maximum-entropy unfolding are shown in Fig. 4 and Fig. 5, with the group-wise, relative error shown in Fig. 6. The four cases led to average, relative, groupwise errors of $201 \%, 126 \%, 63 \%$, and $51 \%$, respectively. The rather simple algorithm (with no prior information), is able to produce reasonably close spectral shapes with as few as 5 responses. It was observed in scoping studies that inclusion of ${ }^{238} \mathrm{Pu}$ has an important effect on the unfolding process. In particular, and as can be observed in Fig. 6, the error between groups 30 and 45 (or energies from 0.78 to $2.1 \mathrm{eV}$ ) is reduced substantially with inclusion of ${ }^{238} \mathrm{Pu}$ and its transmutation precursor, ${ }^{237} \mathrm{~Np}$. However, errors remain in this energy range and lower energies, which indicates that additional, thermalsensitive information (e.g., from thermal filters like Cd) may be of use.

Recall that (6) implies that the total flux is known as part of the maximum entropy unfolding process. However, the total flux may not be known, or it may be known only imprecisely. To understand how the total flux constraint impacts the unfolded spectrum, case 4 was solved using three different total fluxes: 1.0, 0.5, and 2.0. For all three cases, a flux normalized to 1.0 was used to generate the responses. In other words, for a total flux of 0.5 and 2.0, (1) and (6) used inconsistent fluxes, as would be the case were the total flux not known. Shown in Fig. 7 are the group-wise, relative errors resulting from the study. On the average, if the total flux is doubled or halved (relative to the flux used for generating responses), the resulting average, group-wise error is less than a factor of three. In other words, the unfolding is not extremely sensitive to the total flux normalization.

The original scope of this study intended to focus on the simplest of maximum entropy techniques. However, a comparison to simple inversion techniques is warranted. Shown in Fig. 8 are the results for case 4 based on maximum entropy,

\footnotetext{
${ }^{1}$ Note, all results and figures were computed using Python (3.6.0) with NumPy (1.11.3), SciPy (0.18.1), and Matplotlib (2.0.0). All code to generate the figures and this document are available at https://github.com/robertsj/ fission_chamber_spectrometer.
}

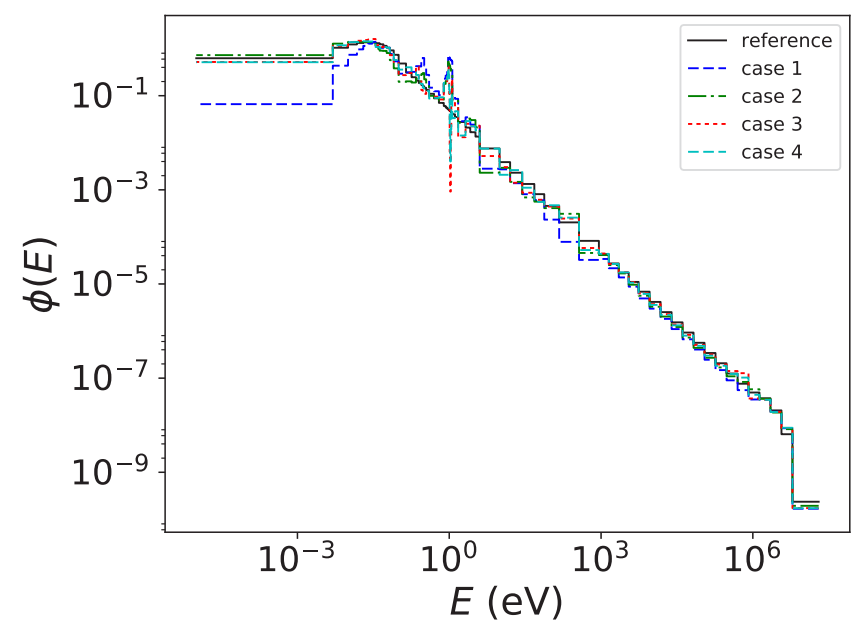

Fig. 4. Unfolded, multigroup spectra for cases $1-3$ with reference spectrum for comparison.

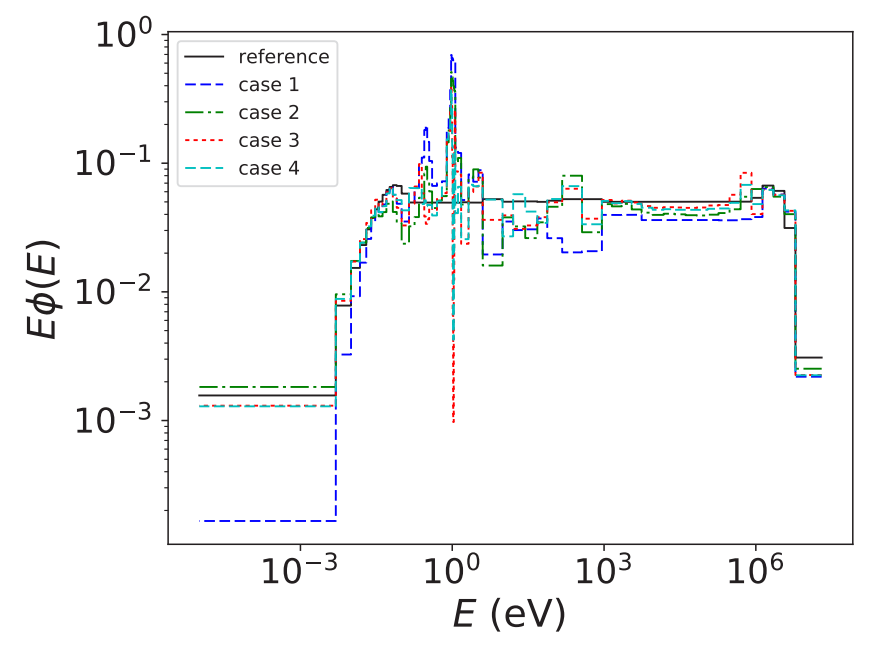

Fig. 5. Same as Fig. 4 but per unit lethargy.

minimum-norm, and Tikhonov-regularization. For the latter, two values of $a$ were used: 0.1 and 0.01 . Overall, the maximumentropy spectrum is closer (qualitatively and quantitatively) to the reference spectrum. Both the minimum-norm and Tikhonovregularization solutions contained negative values at energies near $1 \mathrm{eV}$. Finally, the Tikhonov-regularization solution appears to be rather insensitive to the value of $a$ used in this particular application.

Inspired by other work presented at this conference [9], a study was performed to determine the utility of thermal-neutron filters for providing additional responses given a fixed number of fissile nuclides. In particular, case 3 was revisited with the addition of ${ }^{113} \mathrm{Cd}$ and ${ }^{155} \mathrm{Gd}$. As a simple approximation, the attenuation of thermal neutrons due to $0.025 \mathrm{~mm}$ foils of each isotope was built directly into the fission response functions, the results of which are shown in Fig. 9 and Fig. 10. The filters were only applied to the nuclides with appreciable thermal 


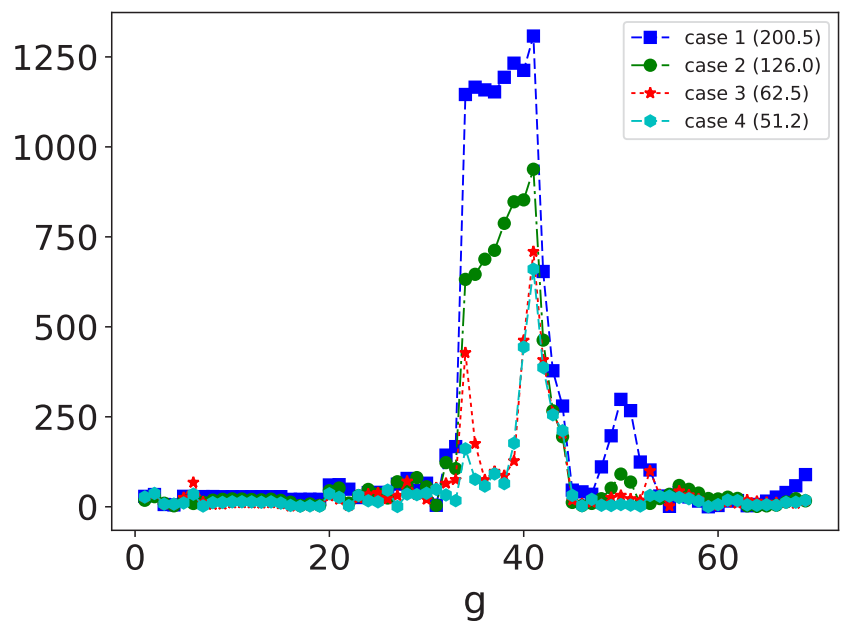

Fig. 6. Group-wise, relative errors (\%). Average error shown in parentheses inside the legend.

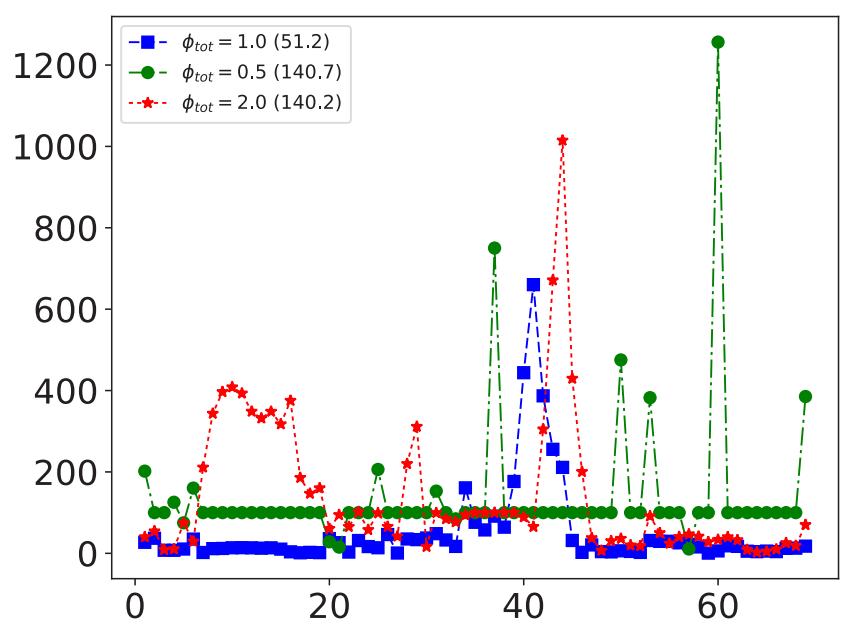

Fig. 7. Impact of total flux normalization on group-wise, relative errors (\%) for case 4. Average errors shown in parentheses inside the legend.

sensitivity, i.e., ${ }^{233} \mathrm{U},{ }^{235} \mathrm{U},{ }^{238} \mathrm{Pu},{ }^{239} \mathrm{Pu}$, and ${ }^{241} \mathrm{Pu}$.

The results of the filter study are shown in Fig. 11, with the results near $1 \mathrm{eV}$ magnified in Fig. 12. The results indicate that filtering improves the results, with use of $\mathrm{Cd}$ leading to a reduction from $63 \%$ to $39 \%$ in the average, group-wise, relative error. Use of $\mathrm{Gd}$ is less effective, reducing the error to $53 \%$. A combination of both filters leads to nearly a reduction by two in the error. Note, although only the same number of fissile nuclides were used in this scenario, the addition of one filter doubles the number of unique responses available, while two filters triples the number of responses.

\section{CONCLUSION}

The primary question addressed by this work has been whether or not a multichambered fission detector could be used for in-core spectroscopy. The basic conclusion to be

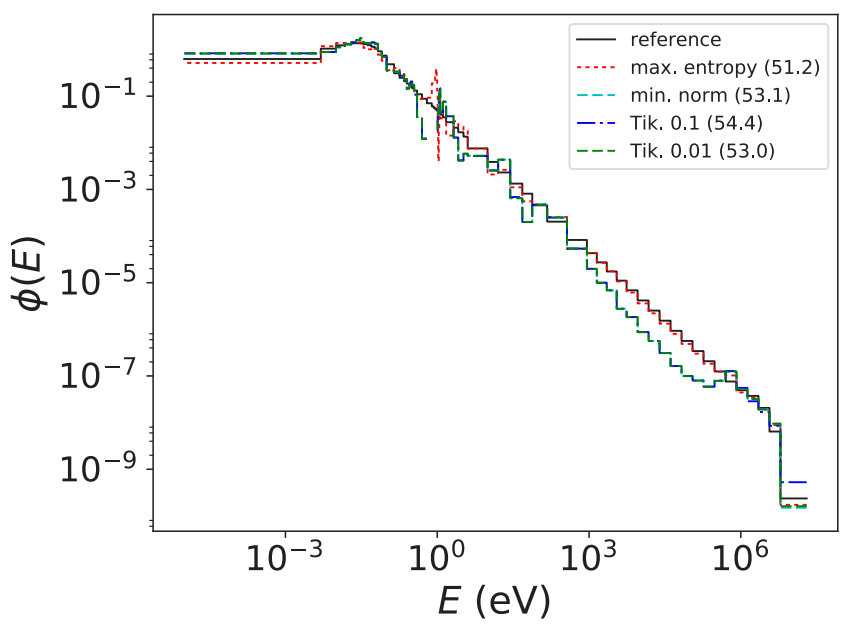

Fig. 8. Comparison of maximum-entropy, minimum-norm, and Tikhonovregularization spectra for case 4 . Average errors (\%) shown in parentheses inside the legend.

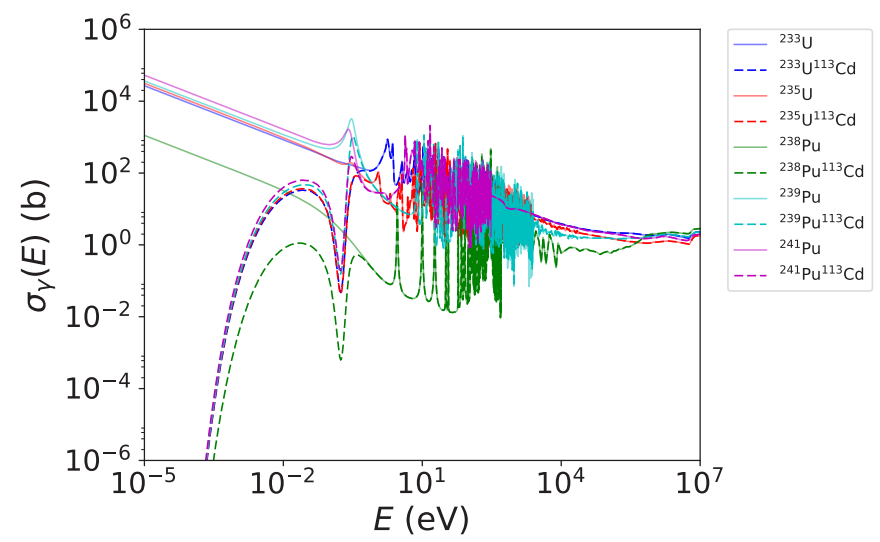

Fig. 9. Cd-filtered and unfiltered responses for thermal-sensitive nuclides of case 3 .

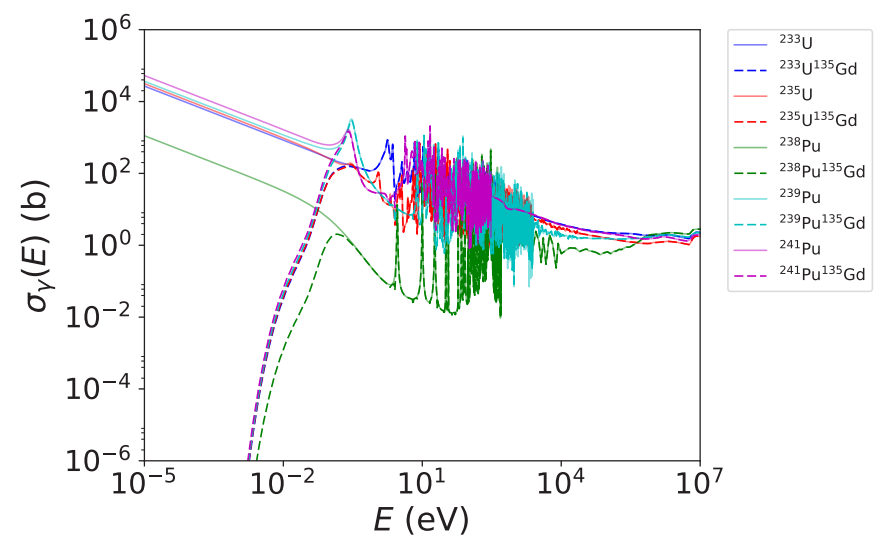

Fig. 10. Gd-filtered and unfiltered responses for thermal-sensitive nuclides of case 3 . 


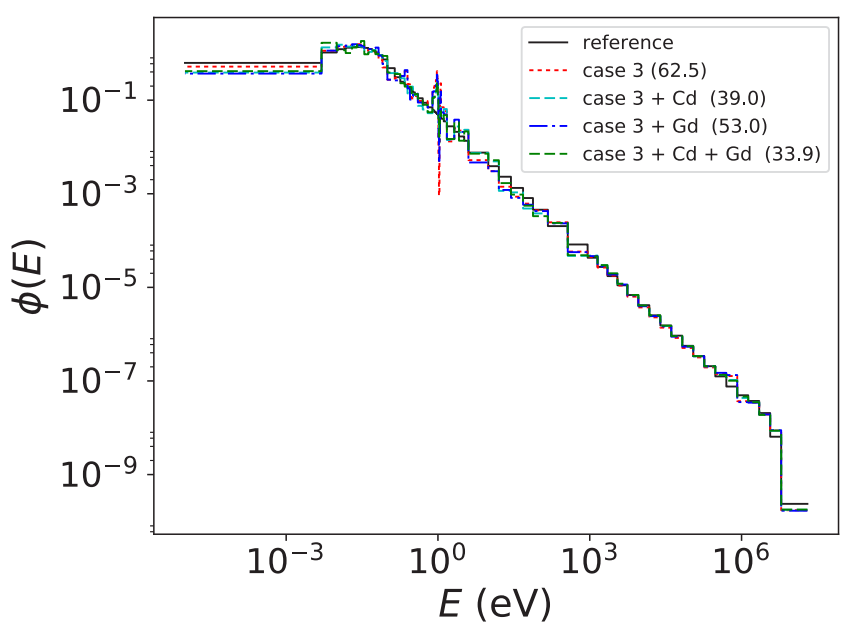

Fig. 11. Unfolded spectra for case 3 with and without filters. Average errors (\%) shown in parentheses inside the legend.

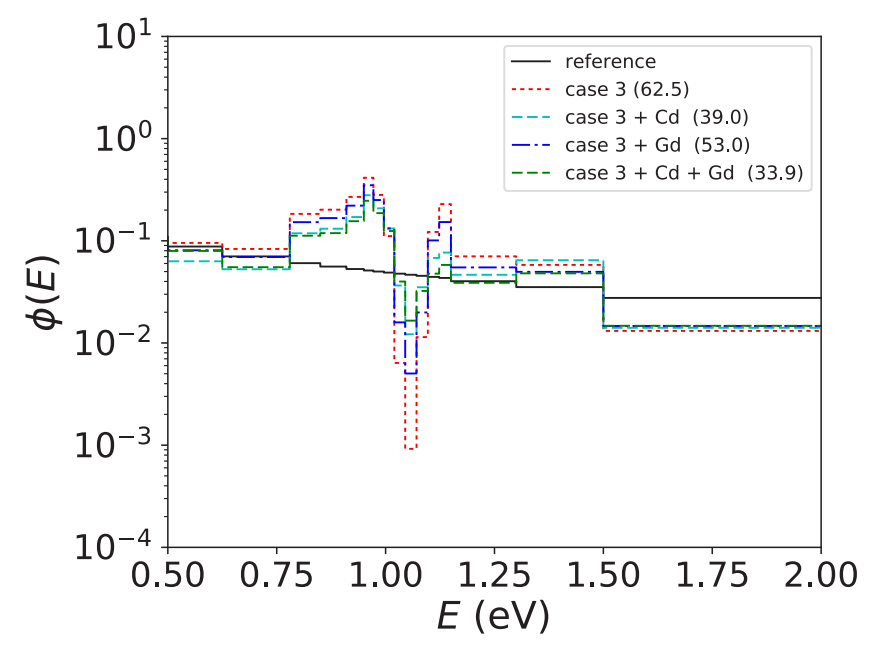

Fig. 12. Same as Fig. 11 but zoomed in on energies near $1 \mathrm{eV}$.

made is that such spectroscopy is possible, to an extent. Overall, the maximum entropy approach, when coupled to 5 or more responses (whether from unique nuclides or use of filters), can provide spectra with average, group-wise, relative errors of less than $100 \%$ assuming that the total flux is well known.

Clearly, this work is scoping in nature, and the algorithms used throughout are simple and incorporate no prior information. More complicated methods should be explored that admit uncertainties in the responses and use of prior spectral information, e.g., like those implemented in the MAXED unfolding code [10], [11]. In addition, more work should be performed to identify candidate filters to maximize the utility of small subsets of fissile (or fissionable) nuclides given that the availability of some nuclides included in this study may be limited.

\section{ACKNOWLEDGMENT}

The author would like to thank Prof. Amir Bahadori for some helpful discussions.

\section{REFERENCES}

[1] C. Blandin, S. Breaud, L. Vermeeren, and M. Weber, "Development of New Sub-miniature Fission Chambers: Modelling and Experimental Tests," Progress in Nuclear Energy, vol. 43, no. 1, pp. 349-355, 2003.

[2] D. S. McGregor, M. F. Ohmes, R. E. Ortiz, A. S. Ahmed, and J. K. Shultis, "Micro-pocket Fission Detectors (MPFD) for In-Core Neutron Flux monitoring," Nuclear Instruments and Methods in Physics Research Section A: Accelerators, Spectrometers, Detectors and Associated Equipment, vol. 554, no. 1, pp. 494-499, 2005.

[3] M. A. Reichenberger, T. C. Unruh, P. B. Ugorowski, T. Ito, J. A. Roberts, S. R. Stevenson, D. M. Nichols, and D. S. McGregor, "Micro-Pocket Fission Detectors (MPFDs) for In-Vore Neutron Detection," Annals of Nuclear Energy, vol. 87, pp. 318-323, 2016.

[4] J. K. Shultis, "Determining Axial Fuel-Rod Power-Density Profiles from In-Vore Neutron Glux Measurements," Nuclear Instruments and Methods in Physics Research Section A: Accelerators, Spectrometers, Detectors and Associated Equipment, vol. 547, no. 2, pp. 663-678, 2005.

[5] "National Nuclear Data Center," https://www.nndc.bnl.gov/, accessed: 2017-07-03.

[6] "Methods of steady-state reactor analysis in nuclear design."

[7] J. L. Mueller and S. Siltanen, Linear and Nonlinear Inverse Problems with Practical Applications. SIAM, 2012.

[8] S. Itoh and T. Tsunoda, "Neutron Spectra Unfolding with Maximum Entropy and Maximum Likelihood," Journal of Nuclear Science and Technology, vol. 26, no. 9, pp. 833-843, 1989.

[9] T. Kaiba, V. Radulovic, D. Fourmentel, L. Barbot, C. Destouches, G. Zerovnik, and L. Snoj, "Calculations to Support to Online Neutron Spectrum Adjustment by Measurements with Miniature FissionChambers in the JSI TRIGA Reactor," in Proc. of ANIMMA 2017.

[10] M. Reginatto and P. Goldhagen, "MAXED, a Computer Code for Maximum Entropy Deconvolution of Multisphere Neutron Spectrometer Data." Health Physics, vol. 77, no. 5, pp. 579-583, 1999.

[11] M. Reginatto, P. Goldhagen, and S. Neumann, "Spectrum Unfolding, Sensitivity Analysis and Propagation of Uncertainties with the Maximum Entropy Deconvolution Code MAXED," Nuclear Instruments and Methods in Physics Research Section A: Accelerators, Spectrometers, Detectors and Associated Equipment, vol. 476, no. 1, pp. 242-246, 2002. 